\title{
Soil Sampling of Burkholderia pseudomallei in and around Mangalore, India - an Explorative Study
}

\author{
Chandrakar S* and Dias M \\ Department of Microbiology, Father Muller Medical \\ College, India \\ *Corresponding author: Sagar Chandrakar, \\ Department of Microbiology, Father Muller Medical \\ College, India
}

Received: December 02, 2016; Accepted: March 15, 2017; Published: March 21, 2017

\begin{abstract}
Introduction: Melioidosis is an infectious disease of public health importance caused by a saprophytic Gram-negative bacterium and a Tier 1 select agent, Burkholderia pseudomallei found in soil and water. It is endemic in countries in Southeast Asia including the Indian subcontinent and in northern Australia. Several cases have been reported across India but no attempt has been made to explore the habitat of Burkholderia pseudomallei from soil and water.
\end{abstract}

Aims: To determine the presence or absence of Burkholderia pseudomallei in the soil in Mangalore and its characterization using conventional and molecular methods.

Settings and Design: A prospective field study carried out for three seasons (Between November 2012 - August 2013).

Methods and Materials: In an exploratory study, soil samples were collected from multiple sites through three seasons (summer, winter, monsoon) systematically based upon presence of positive Melioidosis cases in the vicinity and screened for Burkholderia pseudomallei by culture methods and biochemical reactions. Isolated bacteria were confirmed later by latex agglutination test, $16 \mathrm{~S}$ rDNA and TTS-1 (Type Three secretion system) Polymerase Chain Reaction (PCR) and MALDI-TOF mass spectrometry (Bruker).

Results: Out of samples collected from ten sites through three seasons, soil sample collected from one site post monsoon (winter) yielded Burkholderia pseudomallei.

Conclusion: We describe the first isolation of Burkholderia pseudomallei from the soil in western coastal region of India which will pave the path for future studies to describe the geographical niche and environmental factors affecting the Burkholderia pseudomallei distribution.

Keywords: Burkholderia pseudomallei; Mangalore; India; MALDI TOF; PCR; Soil

\section{Abbreviations}

16SrDNA: 16subunit Ribosomal Deoxyribonucleic Acid; BP: Base Pair; B. pseudomallei: Burkholderia pseudomallei; DRDE: Defence Research \& Development Establishment; DNA: Deoxyribonucleic Acid; HOD: Head of Department; ICMR: Indian Council of Medical Research; MALDI TOF: Matrix-Assisted Laser Desorption/ Ionization- Time of Flight; mm: Millimeters; PCR: Polymerase Chain Reaction; TBSS-C50: Threonine Basal Salt Plus Colistin 50 mg/L; TTSS: Type Three Secretion System; VNBC: Viable But Non Culturable.

\section{Introduction}

Burkholderia pseudomallei, is a soil dwelling saprophyte and a causative agent of Melioidosis. It is found in environment much across south East Asia and northern Australia and increasing numbers being reported from new clusters including parts of South America [1]. Melioidosis occurs as a result of activities leading to increased exposure to environments containing $B$. pseudomallei. The route of infection may be through direct skin inoculation or contamination of wounds; although rarely transmission by inhalation and ingestion has also been reported [2]. Clinical spectrum ranges from a subclinical presentation to full blow septicaemia with fulminant septic shock with mortality up to $90 \%$ [3]. Due to its increased resistance, high mortality and potential aerosol transmission, it is being upgraded from Select B agent to Tier 1 Select Agent group, a group restricted for exceptionally virulent and dangerous pathogens [4].

B. pseudomallei have been commonly isolated from soil and water samples in the endemic areas of Thailand and northern Australia [5-8]. However, the geographical distribution of B. pseudomallei is still very unclear. Several positive melioidosis cases have been reported across India mainly from the western coastal Konkan belt of Karnataka despite which B. pseudomallei has never been isolated from soil or water samples in India [9]. Studies have shown a positive correlation between incidence of melioidosis and monsoon rains [2]. Environment studies have found increased melioidosis cases among paddy workers [10]. Citing the presence of similar environmental
J Bacteriol Mycol - Volume 4 Issue 2 - 2017

Chandrakar et al. (C) All rights are reserved
Citation: Chandrakar S and Dias M. Soil Sampling of Burkholderia pseudomallei in and around Mangalore, India - an Explorative Study. J Bacteriol Mycol. 2017; 4(2): 1046. 
Table 1: Temperature and relative humidity on soil sampling collection dates.

\begin{tabular}{|c|c|c|c|c|c|c|c|}
\hline & & 17 Nov & 18 Nov & 20 April & 21 April & 20 July & 21 July \\
\hline $\begin{array}{c}\text { Temperature } \\
\left({ }^{\circ} \mathrm{C}\right)\end{array}$ & Max & 23.1 & 21.3 & 31.6 & 32.4 & 27.5 & 28.9 \\
\cline { 2 - 8 } & Min & 8.7 & 6.4 & 24.6 & 23.9 & 24.2 & 24.8 \\
\hline $\begin{array}{c}\text { Relative } \\
\text { humidity }\end{array}$ & $71 \%$ & $74 \%$ & $68 \%$ & $72 \%$ & $87 \%$ & $89 \%$ \\
\hline
\end{tabular}

conditions such as temperature, precipitation and similar working culture, an environmental soil sampling was carried out to determine the presence of $B$. pseudomallei in order to identify the geographical distribution and map the risks it poses to humans and livestock [6].

Lately, there is an increase surge of reports of melioidosis cases from Mangalore and surrounding areas during the monsoon rains $[9,11]$. Hence this study is being taken up to record the influence of seasonal variation on its occurrence in the soil which may help in determining the magnitude of problem in Mangalore and surrounding areas. Soil sampling is important to identify the geographic areas where humans and animals are at risk of exposure and that aim of the study was achieved.

\section{Methodology}

\section{Sampling method}

In an exploratory study, soil samples were collected from ten different sites in Mangalore $\left(12.87^{\circ} \mathrm{N}, 74.88^{\circ} \mathrm{E}\right)$, a port town of Dakshina Kannada district, on the western coast of Karnataka, India (Figure 1). Since the study was carried out in an area where the presence/distribution of the B. pseudomallei was unknown, a pilot study was carried out with the sampling sites allocated based on the positive melioidosis cases documented over last two years. 50 soil samples were collected each for three consecutive seasons: Winter (November - January), Summer (February - May), Monsoon (June - October). First lot of soil sample collection was carried on 17 - 18 Nov 2012 followed by second on 20 - 21 April 2013 and third on 20 -
21 July 2013. The soil samples were collected within 100 meters from around household and working area, depending whether person was working outdoor or working from home such as in case of farmers or agriculturist, the soil was collected from the paddy fields whereas those with sedentary work, the soil was collected from around the household. No specific permissions were required for these locations/ activities as it did not involve any private land or any protected land.

\section{Sampling strategy}

Each sampling site was marked and divided in the form of a square grid of $5 \mathrm{~m} \times 5 \mathrm{~m}$. A total of 5 samples were taken from each of the ten sites. Samples were taken from each corner of the grid and last sample from centre of the grid, such that internal spacing between the samples was 3.5 - 5 meters.

\section{Soil sampling}

Soil was collected from a depth of $30 \mathrm{~cm}$ from each sampling point, using a clean spade and from the bottom of the hole about $100 \mathrm{gms}$ of soil will be collected in sterile whirl-pack bags (Hi-media) to avoid contamination. Samples were stored at ambient temperature and transported to the laboratory for same day processing. The spade was cleaned in between each use and rinsed with water to remove any visible debris and then $70 \%$ ethanol prior to next collection of sample. All soil samples were collected in between 9am - 3pm. The type of soil collected was determined as sandy or clay depending on (\% of sand, loam and clay). Further humidity, precipitation, and temperature on the day of sample collection were also considered (Table 1). Samples were collected from the same site on all three seasons.

\section{Processing and isolation}

To each sample containing $100 \mathrm{grams}$ of soil, $100 \mathrm{ml}$ of sterile water was added and mixed by manual shaking. It was allowed to settle overnight and from the upper liquid layer $1 \mathrm{ml}$ was transferred to a sterile container with $9 \mathrm{ml}$ of Ashdown broth containing 50mg/l of

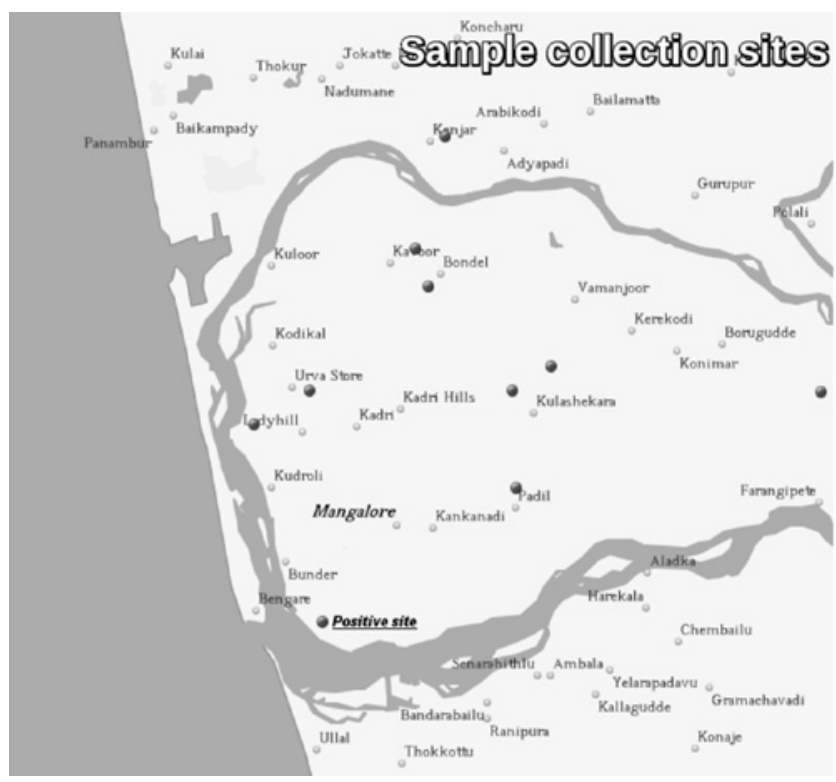

Figure 1: Soil Sampling Sites Ten different locations of soil sampling based on previous melioidosis cases in the father Muller Medical college catchment area Mangalore. The location of sampling site along with the positive site is indicated in the map. Soil sampling was carried out through three seasons (summer, monsoon and winter). 


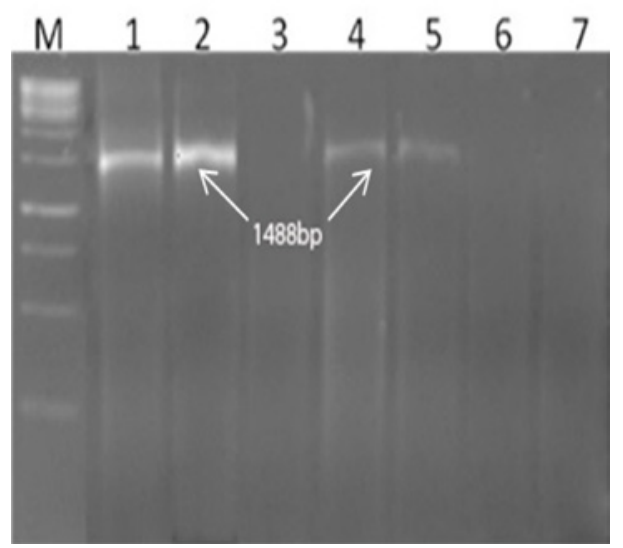

Figure 2: 16S rDNA PCR for confirmation- Lane M: DNA Ladder, Lane 1 \& 2: B. pseudomallei NTCC 1688, Lane 3: Negative control, Lane 4 \& 5: Soil isolate.

colistin and crystal violet. It was incubated at $40^{\circ} \mathrm{C}$ in air for 48 hours after which $10 \mu \mathrm{l}$ was plated and streaked over the Ashdown agar so as to achieve single isolated colonies [12]. The plate was incubated at $40^{\circ}$ $\mathrm{C}$ in air and inspected after every 24 hours for next 7 days. Colonies of $B$. pseudomallei were identified by colony morphology which includes characteristic purple, flat, dry and wrinkled cauliflower like growth colonies on Ashdown agar plate.

\section{Biochemical and Phenotypic Confirmation}

Colonies were suspected to be B. pseudomallei if oxidase positive, resistant to colistin and gentamicin but susceptible to amoxicillinclavulanic acid, di hydrolyses arginine and is unable to assimilate arabinose [13]. Positive colonies were confirmed using highly specific latex agglutination test (An in-house preparation; positive for $B$. pseudomallei but negative for B. thailandensis) [14].

\section{Molecullar Confirmation}

The isolate was further confirmed by identification using PCR based procedures based on amplification of $16 \mathrm{~S}$ rDNA gene (Figure 2 ) and the putative virulent determinant TTSS gene (Figure 3 ) and MALDI-TOF analysis [14] (Table 2).

\section{Results}

A total of 150 samples were processed over a period of three seasons (50 samples each season). 108 out of 150 were suspected to be $B$. pseudomallei based upon characteristic colony morphology and growth on Ashdown agar after incubation at $40^{\circ} \mathrm{C}$ for $48-72$ hours. Conventional Biochemical profiling for each isolate was been carried out. Only one isolate collected on 18 Nov 2012 matched completely with $B$. pseudomallei biochemical profile with negative arabinose sugar assimilation. The antibiotic susceptibility pattern was consistent

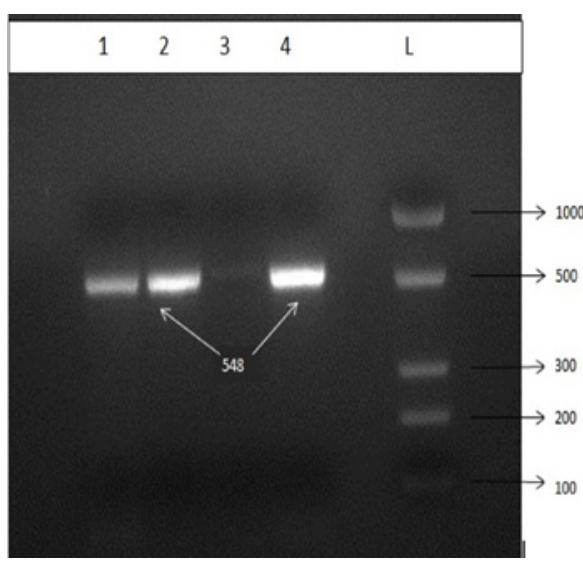

Figure 3: TTSS PCR for confirmation- Lane L: DNA ladder, Lane 1 \& 2: Soil isolate, Lane 3: Negative control, Lane 4: Positive control (A confirmed isolate of Burkholderia pseudomallei).

when tested against antibiotics, colistin $(25 \mathrm{mcg} / \mathrm{disk})$, gentamicin (10mcg/disk) and Amoxicillin-clavulanate (20/10mcg/disk). Isolates from soil sample taken from same site was negative for $B$. pseudomallei growth during the summer and monsoon. The isolate was further processed for molecular confirmation and was found positive by $B$. pseudomallei specific PCR for the 16S rDNA and TTSS gene (TTSS PCR) with the amplification of $1,488 \mathrm{bp}$ and $548 \mathrm{bp}$ respectively. The MALDI-TOF spectrum analysis was carried out using Microflex III mass spectrometer (Bruker Daltonik) and data was imported into Bio Typer software, version 3.0 (Bruker Daltonik GmbH, Bremen, Germany), and was analyzed by standard pattern matching against the 4110 mass spectra of different bacterial species as reference from the Biotyper database which generated the Burkholderia pseudomallei species score (2.14) confirming genera and species.

\section{Discussion}

Burkholderia pseudomallei are an environmental saprophyte found in soil and water. It is known for causing melioidosis, a disease of public health importance in both South East Asia and Northern Australia, where it is endemic and associated with high mortality rates. Infection is not restricted to humans only but a wide variety of animals are susceptible to melioidosis, but some are considered relatively resistant despite their constant exposure to mud [2].

Ours was a first environmental study been carried out in western coastal region of India, defining the geographical distribution of $B$. pseudomallei in the soil. It was being carried out to detect presence or absence of $B$. pseudomallei in the soil in Mangalore, a town in the Dakshina kannada district of India.

The association between melioidosis and soil is supported by increased incidence during monsoon and in those with occupational

Table 2: Primers used in molecular confirmation study.

\begin{tabular}{|c|c|c|c|c|}
\hline Primer name & Primer sequences & Annealing temperature & Amplification size & $\begin{array}{l}\text { Purposel } \\
\text { Reference }\end{array}$ \\
\hline F8 & 5' AGTTTGATCCTGGCTCAG 3' & \multirow{2}{*}{$50^{\circ} \mathrm{C}$} & \multirow{2}{*}{$1,488 \mathrm{bp}$} & \multirow{2}{*}{ Identification [15] } \\
\hline R1492 & 5' ACCTTGTTACGACTT 3' & & & \\
\hline BPTTS1-F & 5'CGTCTCTATACTGTCGAGCAATCG 3' & \multirow{2}{*}{$58^{\circ} \mathrm{C}$} & \multirow{2}{*}{$548 \mathrm{bp}$} & \multirow{2}{*}{ Identification [16] } \\
\hline BPTTS1-R & 5' CGTGCACACCGGTCAGTATC 3' & & & \\
\hline
\end{tabular}


and recreational exposure to surface water and mud, particularly with flooding of paddy fields or planting of seeds [2]. This part of the country receives very high rainfall and farming is one of the important livelihoods of the people. Working in the paddy fields during rainy season is the order of the day and we have observed an increased incidence of melioidosis cases during monsoon.

Studies have shown association of clay with Burkholderia pseudomallei prevalence since clay is known to be rich in nutrients, water and small pore size allows possible electrostatic interactions with bacteria [18]. Reddish and yellowish coloured soil indicates rich presence of oxidized iron compounds which further support of $B$. pseudomallei in clay [19]. The soil in the district is mostly lateritic type, found distributed in the Pediplain area characterised by high iron and aluminium content. Under the Köppen climate classification, Mangalore has a tropical monsoon climate and is under the direct influence of the Arabian Sea branch of the southwest monsoon. It receives about 95 per cent of its total annual rainfall within a period of about six months from May to October, while remaining extremely dry from December to March [20].

As all the environmental factors are favourable for the growth of bacteria in this area, we tried to detect the bacteria from the soil. In our study, all the soil sampling sites were residential sites which are part of urban Mangalore. Thus, the history of land usage could not be obtained as it underwent disturbances due to human or natural causes. The $\mathrm{pH}$ of the soil collected from different sites varied little between the seasons being more acidic ( 4.4 - 4.7 ) during summers to less acidic (4.8 - 5.4) during monsoons. Of the ten sites, only one site showed a positive soil isolate of $B$. pseudomallei. The site of soil collection was located about 70 meters from where the clinical melioidosis was being reported in 2011. The isolate found was positive during the month of November when the monsoons have regressed and soil is saturated with water. The $\mathrm{pH}$ of the soil turns more towards neutral as the salinity decreases and excessive rains raises the water table bringing the bacteria to upper soil layers [21]. All these environmental factors provide favourable conditions for survival and propagation in the soil which could be the reason for isolation of the organism from the soil sample. In the month of April, due to increased temperature the soil becomes dried up, cracked and is subjected to salinization conditions which do not support the survival [21]. In July, the monsoon is at the peak with average rainfall of greater than $1000 \mathrm{~mm}$ resulting in surface wash off the soil layers and this might be the reason samples collected during monsoon were negative.

B. pseudomallei have got higher affinity for the acidic environment. Presence of animals reduces the probability of isolation due to nitrification process of animal urine derived ammonium which increases the $\mathrm{pH}$ [22]. This may be another contributing reason for not isolating many bacilli from the samples in our study

Due to paucity of resources and absence of clinical isolates, comparison of soil and clinical sequences from the study site was not possible which could have provided more details and insights into the findings and in epidemiological typing.

A study carried out on surface water during monsoons and paddy fields in the area for isolation of $B$. pseudomallei might yield positive results due to favourable environmental and topographical conditions.
Though we got only one positive isolate from 150 samples collected over a period ranging various seasons, it is a significant finding, but more samples need to be tested to record the exact magnitude of the problem.

\section{Limitations of the Study}

Insufficient numbers of soil samples were taken from a single site, which runs the risk of low power of detection and a high false negative (Type II error) rate; this can be avoided by increasing number of samples (100 samples per site) taken. Ashdown medium was used for isolation and identification which has lower sensitivity for detection. Threonine basal salt plus colistin $50 \mathrm{mg} / \mathrm{L}$ (TBSS-C50) is a better alternative and has higher sensitivity. Furthermore, isolation based upon culture colonies may be misleading as under undue stress such as Low $\mathrm{pH}$, Osmotic stress the cells turn Viable But Non Culturable (VBNC). Although confirmation of the isolate was carried out using molecular techniques, detection of $B$. pseudomallei directly from the soil sample using Polymerase chain reaction against TTSS which detects even the DNA of dead bacterium will be more suitable for epidemiological surveillance.

\section{Conclusion}

This is the first published environmental study done in the western coastal part of India. The results of this study indicate the presence of Burkholderia pseudomallei in soil. Molecular technique such as PCR against TTSS is key for Burkholderia pseudomallei identification. Our findings will pave way for the future environmental studies for the distribution of $B$. pseudomallei from this area, and the effect of environmental factors such as water content, soil type, $\mathrm{pH}$ and vegetation on the habitat of $B$. pseudomallei.

\section{Acknowledgement}

We are thankful to Indian Council of Medical Research (ICMR) for providing fund for the study. We are also thankful to Dr. Duraipandian Thavaselvam, Division of Microbiology, DRDE, Gwalior, India for carrying out molecular characterization and Bio typing and Dr. Chiranjay Mukhopadhyay, Professor and HOD, Department of Microbiology, Kasturba Medical College, Manipal, India for aiding with latex agglutination and Polymerase Chain Reaction against TTSS of Burkholderia pseudomallei.

\section{References}

1. Currie BJ, Dance DA, Cheng AC. The global distribution of Burkholderia pseudomallei and melioidosis: an update. Trans R Soc Trop Med Hyg. 2008; 102: S1-S4.

2. Cheng AC, Currie BJ. Melioidosis: epidemiology, pathophysiology, and management. Clin Microbiol Rev. 2005; 18: 383-416.

3. White NJ. Melioidosis. The Lancet. 2003; 361: 1715-1722.

4. Butler D. Viral research faces clampdown. Nature. 2012; 490: 456

5. Limmathurotsakul D, Wongsuvan G, Aanensen D, Ngamwilai S, Saiprom $\mathrm{N}$, Rongkard $\mathrm{P}$ et al. Melioidosis caused by Burkholderia pseudomallei in drinking water, Thailand, 2012.Emerg Infect Dis. 2014; 20: 265-268.

6. Kaestli M, Mayo M, Harrington G, Ward L, Watt F. Hill JV et al. Landscape changes influence the Occurrence of the Melioidosis Bacterium Burkholderia pseudomallei in Soil in Northern Australia. PloS Negl Trop Dis. 2009; 3: 364.

7. Wuthiekanun V, Limmathurotsakul D, Chantratita N, Feil EJ, Day NP, Peacock SJ. Burkholderia pseudomallei are Genetically Diverse in Agricultural Land in Northeast Thailand. PloS Negl Trop Dis. 2009; 3: 496. 
8. Mayo M, Kaesti M, Harrington G, Cheng AC, Ward L, Karp D et al. Burkholderia pseudomallei in unchlorinated domestic bore water, Tropica Northern Australia. Emerg Infect Dis. 2011; 17: 1283-1285.

9. Vidyalakshmi K, Lipika S, Vishal S, Damodar S, Damodar S, Chakrapani M Emerging clinico-epidemiological trends in melioidosis: analysis of 95 cases from western coastal India. Int J Infect Dis. 2012; 16: 491-497.

10. Suputtamongkol $Y$, Hall AJ, Dance DA, Chaowagul W, Rajchanuvong A, Smith MD et al. The epidemiology of melioidosis in Ubon Ratchantani, northeast Thailand. Int J Epidemiol. 1994; 23: 1082-1090.

11. Mukhopadhyay C, Kaestli M, Vandana KE, Sushma K, Mayo M, Richardson $L$ et al. Short Report: Molecular Characterization of Clinical Burkholderia pseudomallei Isolates from India. Am. J. Trop. Med. Hyg. 2011; 85: 121-123.

12. Ashdown LR. An improved screening technique for isolation of Pseudomonas pseudomallei from clinical specimens. Pathology. 1979; 11: 293-297.

13. Trakulsomboon S, Vuddhakul V, Tharavichitkul P, Na-Gnam N Suputtamongkol $\mathrm{Y}$, Thamlikitkul $\mathrm{V}$. Epidemiology of arabinose assimilation in Burkholderia pseudomallei isolated from patients and soil in Thailand. Southeast Asian J Trop Med Public Health. 1999; 30: 756-759.

14. Duval BD, Elrod MG, Gee JE, Chantratita N, Tandhavanant $S$, Limmathurotsakul $D$ et al. Evaluation of a latex agglutination assay for the identification of Burkholderia pseudomallei and Burkholderia mallei. Am J Trop Med Hyg. 2014; 90: 1043-1046.

15. Karger A, Stock R, Ziller M, Elschner MC, Bettin B, Melzer F et al. Rapid identification of Burkholderia mallei and Burkholderia pseudomallei by intact cell Matrix-assisted Laser Desorption/Ionisation mass spectrometric typing. BMC Microbiol. 2012; 12: 229.
16. Gee JE, Sacchi CT, Glass MB, De BK, Weyant RS, Levett PN et al. Use of 16S rRNA gene sequencing for rapid identification and differentiation of Burkholderia pseudomallei and Burkholderia mallei. J Clin Microbiol. 2003; 41: 4647-4654.

17. Novak RT, Glass MB, Gee JE, Gal D, Mayo MJ, Currie BJ et al. Development and evaluation of a real time PCR assay targeting the Type III of Burkholderia pseudomallei. J Clin Microbiol. 2006; 44: 85-90.

18. Sylvia D, Fuhrmann J, Hartel P, Zuberer D. Principles and applications of soil microbiology: $2^{\text {nd }}$ edn. New Jersy: Pearson Prentice hall. 2004.

19. Yang HM, Chaowagul W, Sokol PA. Siderophore production by Pseudomonas pseudomallei. Infect Immun. 1991; 59: 776-780.

20. Central Ground Water Board 2012, Ground water information booklet Dakshina kannada district.

21. Inglis TJ, Sagripanti JL. Environmental factors that affect the survival and persistence of Burkholderia pseudomallei. Appl Environ Microbiol. 2006; 72 : 6865-6875.

22. Chen YS, SC Chen, CM Kao, YL Chen. Effects of soil pH, temperature and water content on the growth of Burkholderia pseudomallei. Folia Microbiol. 2003; 48: 253-256.
J Bacteriol Mycol - Volume 4 Issue 2 - 2017
ISSN : 2471-0172 | www.austinpublishing group.com
Chandrakar et al. @ All rights are reserved
Citation: Chandrakar S and Dias M. Soil Sampling of Burkholderia pseudomallei in and around Mangalore, India - an Explorative Study. J Bacteriol Mycol. 2017; 4(2): 1046. 